\title{
Cortical sound processing in children with autism disorder: an MEG investigation
}

\author{
Nicole M. Gage CA , Bryna Siegel,' Melanie Callen' and Timothy P. L. Roberts \\ Biomagnetic Imaging Laboratory, Department of Radiology, Mail Code 5100, 315I Social Sciences Plaza A, University of California, Irvine, \\ CA 92697-5100; 'Pervasive Developmental Disorders Clinic, University of California, San Francisco, CA, USA \\ CACorresponding Author: ngage@uci.edu
}

Received 3 June 2003; accepted I2 June 2003

DOI: 10.1097/0I.wnr.0000090030.46087.4a

\begin{abstract}
Previous work investigating frequency encoding mechanisms in human auditory cortex has provided evidence that latency of the auditory evoked MI00 is strongly proportional to frequency, with low frequency $(100-200 \mathrm{~Hz})$ tones associated with $\sim 30 \mathrm{~ms}$ longer latencies than mid-range frequency $(\mathrm{I}-2 \mathrm{kHz})$ tones. Motivated by pervasive speech and auditory perception deficits observed in autism spectrum disorder, we evaluated MI00 frequency dependence in children with autism disorder and typically developing controls.
\end{abstract}

Results indicate that for control children, the dynamic range of frequency modulation was similar to previous reports for healthy adults. Children with autism had a much reduced range of modulation in right hemisphere sites. Findings indicate that frequency encoding mechanisms may follow a differential maturational path in autism spectrum disorder. NeuroReport 14:2047-2051 (c) 2003 Lippincott Williams \& Wilkins.

Keywords: Auditory cortex; Auditory perception; Autism disorder; Development; Hemispheric asymmetry; Language; Magnetoencephalography; MI00; Neurodevelopmental disorder

\section{INTRODUCTION}

Autism spectrum disorder is a neurodevelopmental disorder diagnosed in 1/500-1/1000 children in the USA each year. Autism is characterized by abnormalities in verbal and non-verbal communication. An early indicator of autism is delay or regression in the development of speech and language in young children [1]. In addition to impaired language, many individuals with autism exhibit unusual sound reactivity [2]. While language deficits and abnormal sound sensitivity are well recognized, and pervasive features of autism, their description is based largely on clinical observations and the etiology is unknown. Language abnormalities couple with atypical sensory sound sensitivity to implicate auditory dysfunction as a potential source of impairment.

In the present investigation, we use MEG to record auditory evoked neuromagnetic responses to simple sounds (sinusoidal tones) in children with autism and in typically developing controls. MEG is well suited for this investigation: it provides submillisecond temporal resoluton of synchronized cortical neural responses and has been demonstrated to be well tolerated by children with autism [3]. This investigation is predicated on earlier investigations in our laboratory with healthy adults, where we observed that the latency of the auditory evoked M100 varies with stimulus attributes such as frequency, acoustic structure, and periodicity [4-6]. For example, in healthy adults, we have observed a precise relationship between the frequency of pure tones and the latency of the M100 component. Specifically, we find that M100 latency is strongly proportional to $1 /$ frequency, with low frequency tones (100$200 \mathrm{~Hz}$, characteristic of the pitch of the human voice) associated with up to $30 \mathrm{~ms}$ longer latencies than mid-range frequencies $(1000-3000 \mathrm{~Hz})$ [7]. A similar finding has been reported for the electric N1, detected by EEG, where the range of latency prolongation for lower frequency $(250 \mathrm{~Hz})$ vs higher frequency $(500-4000 \mathrm{~Hz})$ tones is $\sim 20 \mathrm{~ms}$ in normal hearing adults $[8,9]$. Frequency-induced modulation of the M100 is also evidenced in more complex sounds, where M100 latency reflects secondary spectral features such as harmonic structure and formant frequency [4-6]. Thus, the temporal signature of the M100 (as well as the electric N1) appears to reflect a recoding of spectral properties in sounds into the time domain [7-9]. Measurements of M100 latency modulation as a function of stimulus feature may therefore provide an objective measure with which to assess auditory cortical sensitivity to spectrotemporal features in sounds in both typically developing children and children with neurodevelopmental disorder, such as autism.

Our central hypothesis in the present investigation is that neural sound-processing mechanisms evident in the healthy brain, and which give rise to predictable M100 latency variation as a function of frequency, will not be intact in 
children with autism. To test this hypothesis, we first measure M100 latency modulation as a function of tone frequency in typically developing children and compare results to our previous findings for adults [7]. Since there have been very few MEG studies of either typically developing or neurodevelopmentally disordered children, this investigation is exploratory in nature. Our first aim is to determine if the dynamic range of M100 modulation is similar in typically developing children as compared to normal hearing adults. We have previously reported a dynamic range of M100 latency modulation for tones ranging from 100 to $1000 \mathrm{~Hz}$ of $\sim 25-30 \mathrm{~ms}$ in healthy adults $[4,7]$. Based on previous MEG investigations with children, we expect that, overall, M100 latencies will be somewhat prolonged $(30-70 \mathrm{~ms})$ in children compared with adults $[3,10,11]$. Here we evaluate whether the dynamic range of M100 latency modulation is larger in children then in adults, reflecting an overall expansion of the range commensurate with the prolonged latency found in children, or whether the absolute range is similar in children to previous reports for adults, indicating that the frequency modulation is on a similar scale to adults but shifted in time. Second, we compare results for autism children to controls in order to determine if M100 latency modulation in these children is similar to typically developing controls. Specifically, we evaluate whether the dynamic range of latency modulation reflects a similar sensitivity in typically developing control and children with autism.

\section{MATERIALS AND METHODS}

Participants consisted of 17 (five female) typically developing control children (age 10-16, mean ( \pm s.d.) $13.5 \pm 1.7$ years) recruited from the UC San Francisco community. In addition, 15 male children with autism spectrum disorder (age 8-14, mean $11.4 \pm 2.0$ ) were recruited from the Pervasive Developmental Disorders Clinic at the University of California, San Francisco. Children with autism had normal hearing confirmed by previous audiological assessments available for review in patient charts. Control children had normal hearing as reported by parents or established in previous clinical audiological assessments. Control children were free of known neurological disease. All participants were native speakers of English. All participants were studied without the use of sedation.

Children with autism were diagnosed according to procedures outlined in the California DDS Diagnostic Best Practice for Autism Guidelines [12], including direct observation using a standardized autism-specific behavioral rating, a clinical history designed to rule in autism and rule out related disorders, an age-appropriate cognitive test against which to rate possible autism symptoms versus mental retardation, and finally use of the DSM-IV [13] criteria based on an overall evaluation of these data. The children with autism were selected according to the following inclusion criteria: normal non-verbal or performance IQ (IQ $\geq 70$ as assessed by a version of the Weschler Intelligence Scale for Children (WISC-R or WISC-III) and verbal IQ $\geq 1$ s.d. (15 points) below performance IQ.

MEG scanning required that participants remain motionless for several minutes at a time. In order to increase the likelihood of successful MEG recording, autistic children who met the above-mentioned inclusion criteria were prescreened in an effort to select individuals who would be cooperative during the MEG scanning procedures. Stimulus presentation and MEG recording were performed with the approval of the institutional committee on human research. Informed written consent was obtained from each participant and/or parent.

Stimulus presentation and MEG recordings: Sinusoidal tones of frequency 100, 200, 500, and $1000 \mathrm{~Hz}$ were presented monaurally using Etymotic ER-3A earphones and air tubes designed for use with the MEG system (Etymotic, Oak Brook, IL). Stimuli were presented at $40 \mathrm{~dB}$ SL. Neuromagnetic fields were recorded for each participant using a 37-channel biomagnetometer (MAGNES, BTi, San Diego, CA.) in a magnetically shielded room. The sensor array was placed over the temporal lobe contralateral to the ear of stimulus presentation. Evoked response to a reference $1000 \mathrm{~Hz}$ sinusoidal tone ( $400 \mathrm{~ms}$ duration) was evaluated to determine whether the sensor array was positioned to effectively record the auditory evoked M100 field. Epochs of $600 \mathrm{~ms}$ duration (100 ms pre-stimulus onset and $500 \mathrm{~ms}$ post-stimulus onset) were acquired around each stimulus at a sampling rate of $1041.7 \mathrm{~Hz}$ with a bandwidth of $400 \mathrm{~Hz}$ and a $1.0 \mathrm{~Hz}$ high-pass filter. This procedure was repeated for each hemisphere. Presentation was blocked by stimulus condition. Each stimulus was presented 120 times per block in a passive listening paradigm. Block duration was 2$3 \mathrm{~min}$. Blocks were presented in a pseudorandom order for each of the four stimulus conditions, for each hemisphere. MEG recording continued until each stimulus condition was presented in each hemisphere (for a total of eight scanning blocks) or until the participant was no longer able to tolerate the procedure.

Data analysis: The data were inspected and individual epochs that contained motion-related artifacts $(>2.5 \mathrm{pT})$ were removed. Data were then selectively averaged by stimulus condition and hemisphere for each participant. Averaged waveforms were band-pass filtered using a low cut-off frequency of $1 \mathrm{~Hz}$ and a high cut-off frequency of $40 \mathrm{~Hz}$. The root mean square (RMS) of the field strength across all 37 channels was calculated for each sample point. The M100 peak was determined as the peak in RMS value across 37 channels in the interval $80-200 \mathrm{~ms}$, participant to a single equivalent current dipole (SECD) model/data correlation $r>0.95$, with $\mathrm{Q}<50.0 \mathrm{nAm}$ and a signal-to-noise ratio $\geq 6: 1$. The latency of the M100 component served as the dependent measure.

\section{RESULTS}

Two children with autism were unable to tolerate any portion of the recording process. Auditory evoked neuromagnetic fields were acquired from each of the remaining 13 children with autism and all 17 controls. Signal to noise levels did not reach criterion $(\geq 6: 1)$ in a large percentage (29\% autism, 21\% control) of scans for the lowest frequency tone $(100 \mathrm{~Hz})$ in both groups of children. In order to increase the power of our statistical analyses we excluded the $100 \mathrm{~Hz}$ tone from further date analyses and all subsequent analyses include the 200, 500, and $1000 \mathrm{~Hz}$ tones. 
Previous reports indicate that M100 latency is dependent on age, with longer latencies for younger children [3,10,11]. In order to remove age effects in the present analysis, M100 latency was normalized by dividing M100 latency for each tone by the latency of the $1000 \mathrm{~Hz}$ tone for each hemisphere, for each participant. Normalized M100 latencies > 2s.d. from the mean were removed from subsequent analyses. M100 latency was analyzed using a repeated measures (ANOVA) with group (autism, control) as the betweensubject factor and tone and hemisphere as within-subjects factors. $\alpha=0.05$ was used for all statistical tests.

Frequency dependence of the auditory M100: An initial repeated measures analysis was performed with age as a covariate in order to determine whether the normalization of M100 latencies was effective at removing the effects of age in our two groups of children. Results of this analysis indicated that normalized M100 latencies did not statistically differ as a function of age $(\mathrm{F}(1,13)=0.66, . p=0.43)$. The remaining repeated measures analyses were conducted without age as a co-variate. There was no effect of group $(\mathrm{F}(1,13)=0.24, p=0.63)$; overall, mean ( \pm s.e.m.) normalized M100 latencies were similar across groups (control $1.07 \pm 0.05$; autism: $1.06 \pm 0.04$; Fig. 1 ). A main effect of hemisphere neared statistical significance $(F(1,13)=3.87$, $p=0.07), \mathrm{M} 100$ latencies were generally longer in the left hemisphere $(1.09 \pm 0.06)$ than in the right $1.04 \pm 0.03)$. A main effect of tone was significant $(F(2,26)=15.70$, $p<0.001)$, with longer latencies for the $200 \mathrm{~Hz}$ tone $(1.13 \pm 0.04)$ than for the $500 \mathrm{~Hz}(1.06 \pm 0.04)$ and $1000 \mathrm{~Hz}$ tones (normalized at 1.00).

Figure 2 shows evoked responses from one representative control, with response to the $1000 \mathrm{~Hz}$ tone shown in the upper panel (a) and response to the lower frequency $200 \mathrm{~Hz}$ tone shown in the lower panel (b). This child showed characteristic M100 latency frequency dependence, with peak latency for the lower frequency, $200 \mathrm{~Hz}$ tone (at $123.8 \mathrm{~ms}$ ) somewhat prolonged as compared to the higher frequency $1000 \mathrm{~Hz}$ tone $(113.3 \mathrm{~ms})$. A hemisphere $\times$ tone two-way interaction was significant $(\mathrm{F}(2,26)=3.43, p=0.05$; Fig. 1). No other interactions reached statistical significance.

Dynamic range of M100 latency: age-matched results: The dynamic range of M100 latency modulation by tone frequency was calculated by subtracting latency for the $200 \mathrm{~Hz}$ tone from the $1000 \mathrm{~Hz}$ tone. Our groups varied somewhat in mean age (control 13.5, autism 11.4). In order to better assess between-group differences in the dynamic range of M100 latency by tone frequency, we conducted this portion of the analysis on a subset of control $(n=8)$ and autism $(n=8)$ participants that were closely matched for age (control 12.6 \pm 1.2 ; autism $12.7 \pm 0.9$ ). Because the groups were age-matched, we used un-normalized M100 latencies in the dynamic range analysis. There was no effect of group $(\mathrm{F}(1,11)=0.22, p=0.65)$, although the overall dynamic range was somewhat larger in the autism group $(15.9 \pm 4.7)$ than in controls $(13.2 \pm 5.5)$. The effect of hemisphere did not reach statistical significance $(\mathrm{F}(1,11)=1.84, p=0.20)$; however, the dynamic range was larger in the left $(18.6 \pm 7.7)$ than the right $(10.5 \pm 2.6)$. A hemisphere $\times$ group interaction neared statistical significance $(F(1,11)=3.80, p=0.08)$ :
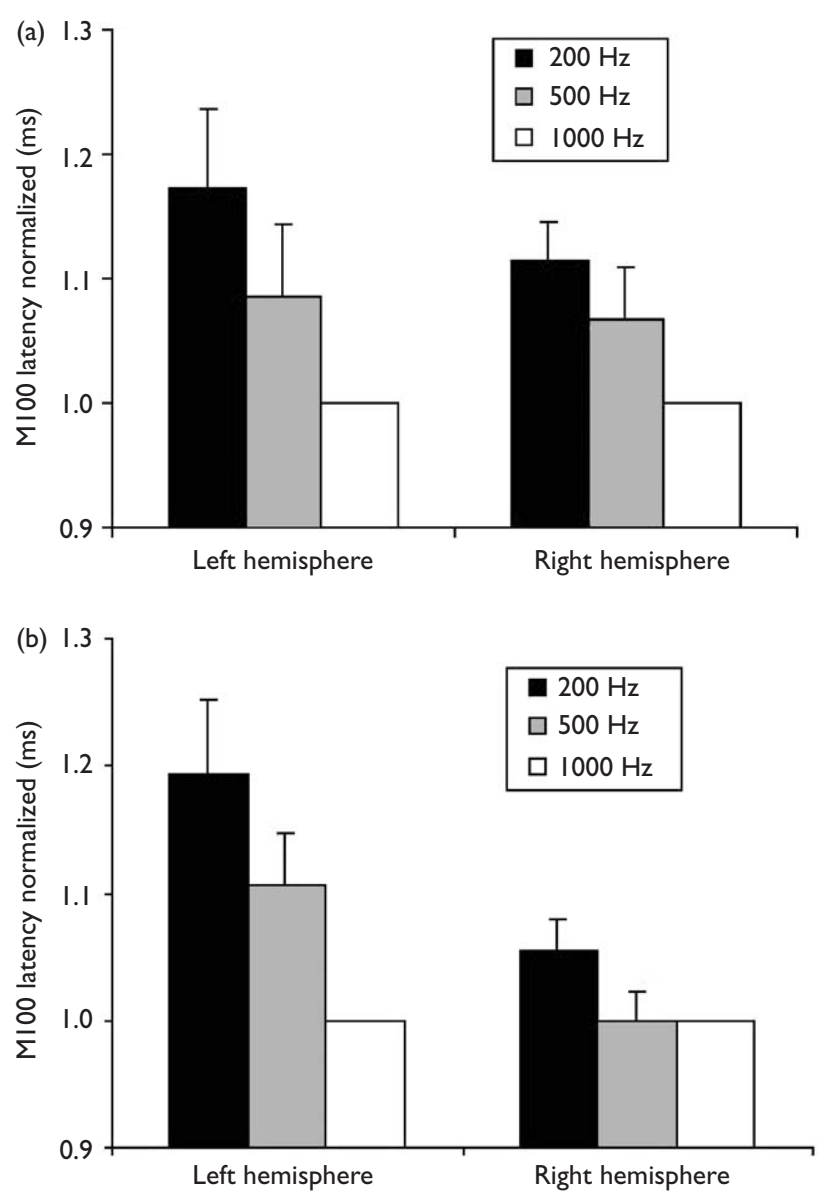

Fig. I. Mean normalized MI00 latency for the 200,500 , and $1000 \mathrm{~Hz}$ tones in the left and right hemispheres of the control group (a) and the autism group (b). Error bars represent s.e.m.

while the dynamic range did not statistically differ by hemisphere for the control group $(t(6)=0.41, p=0.70$; left hemisphere $11.4 \pm 7.6$; right hemisphere $14.9 \pm 3.6$; Fig. 3), it did statistically differ for the autism group $(t(5)=2.48$, $p=0.05$; left hemisphere $25.7 \pm 7.9$; right hemisphere $6.1 \pm 1.6$ ), with a range for the left hemisphere that was $>4$-fold larger than that for the right (see Fig. 3 ).

\section{DISCUSSION}

In the present investigation, we measured the frequency dependence of M100 latency in typically developing and autistic children and report several key observations. First, in the control group, M100 latency differed significantly for each of the 200,500, and $1000 \mathrm{~Hz}$ sinusoidal tones, in each hemisphere (Fig. 1a). In our previous work with healthy adults, we have observed that M100 latency is strongly proportional to 1 / frequency, with a fixed cost of $\sim 100 \mathrm{~ms}$ in the neural processes that lead to the formation of the M100, plus a period dependent time that is equal to roughly three periods of a sinusoid ( $3 \mathrm{~ms}$ for a $1000 \mathrm{~Hz}$ tone, $15 \mathrm{~ms}$ for a $200 \mathrm{~Hz}$ tone) [4]. Our findings reported here for typically developing children, with a dynamic range of latency modulation for the $200-1000 \mathrm{~Hz}$ tones of $10-15 \mathrm{~ms}$ (Fig. 2), 


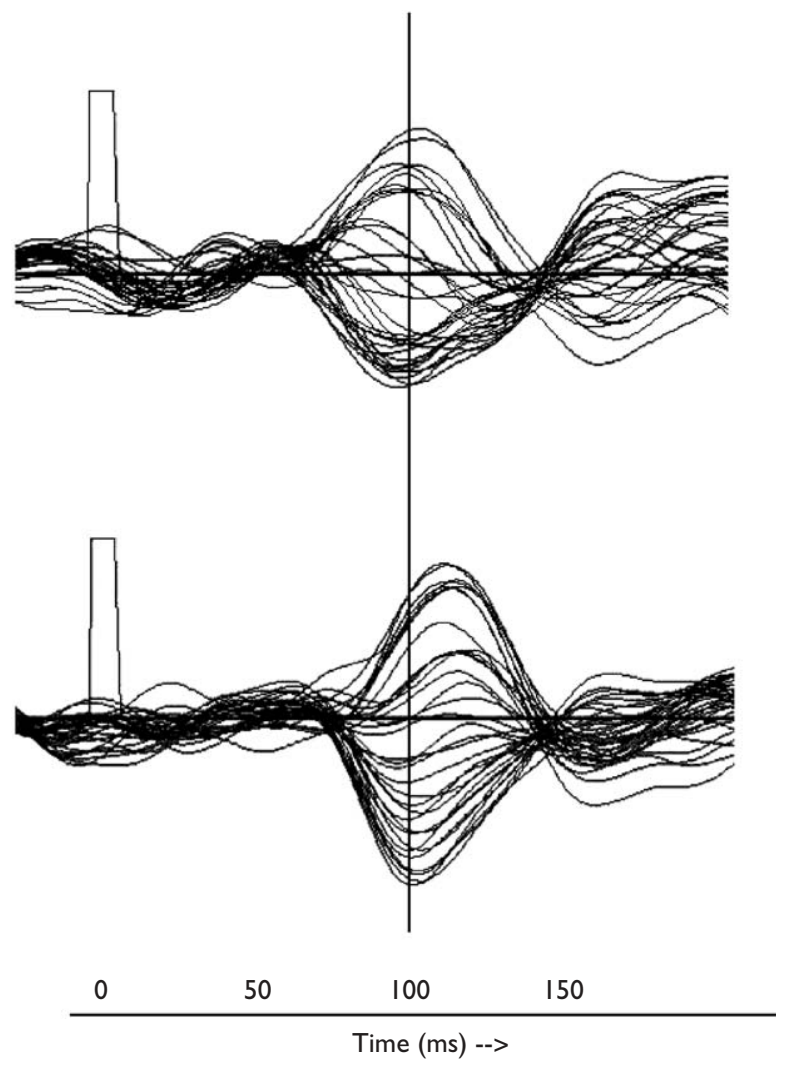

Fig. 2. Auditory evoked neuromagnetic fields recorded over the left hemisphere for one representative control. (a) Response to the $1000 \mathrm{~Hz}$ tone with MI00 latency of III.3 ms; (b) response to the $200 \mathrm{~Hz}$ tone with MI00 latency that is prolonged compared with the higher frequency, at $123.8 \mathrm{~ms}$.

are in good accord with our previous MEG findings for adults [7], as well as findings reported in the EEG literature $[8,9,14]$. Although M100 latency is in general longer in children than in adults, the dynamic range (at 10-15 ms) of M100 modulation by tone frequency in the control group was similar in magnitude to our previous reports for healthy adult. Thus, the absolute magnitude of the dynamic range appears to be shifted in time rather than expanded in scale for children in the age range 10-16 years. Our results for controls imply that the neural mechanisms for coding frequency information in simple sounds may be largely in place and adult-like in typically developing children as early as 10 years of age (the age of the youngest control child studied here).

Second, in children with autism, the M100 latency in the left hemisphere (Fig. 1b) was modulated by tone frequency in a manner that was generally similar to findings for controls in the present investigation, and for healthy adults in our previous study [7]. In the right hemisphere (Fig. 1b), however, we report a different response: while M100 latency statistically differed for the end points of the tones tested $(200-1000 \mathrm{~Hz}$ tone contrast), latencies did not differ for either of the finer-grained contrasts $(200-500 \mathrm{~Hz}$ or 500 $1000 \mathrm{~Hz}$ ). These findings indicate that neural processes that lead to the formation of the auditory M100 may be less sensitive to spectral contrasts in sounds in the right

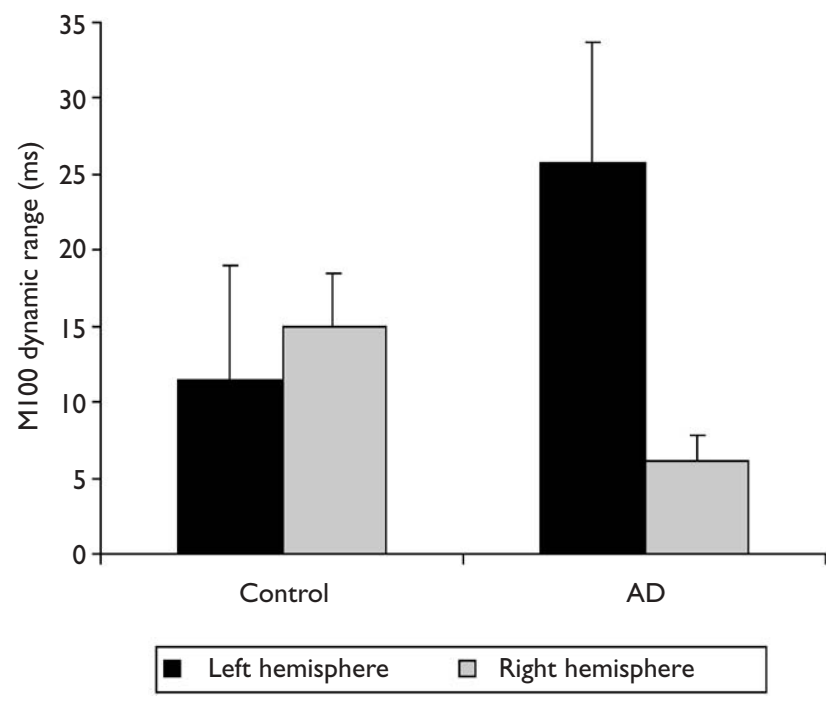

Fig. 3. Dynamic range of MIO0 latency in the left and the right hemispheres for eight control and eight autism age-matched children in response to 200,500 , and $1000 \mathrm{~Hz}$ tones. Error bars represent s.e.m.

hemisphere in children with autism. When we evaluated hemispheric asymmetries in the dynamic range of frequency modulation in closely age-matched groups (control, autism), we found no reliable difference between the range in the left (at $11.4 \mathrm{~ms}$ ) and the right (at $14.9 \mathrm{~ms}$ ) hemispheres in the control group (Fig. 3). In sharp contrast, we report a statistically reliable asymmetry in the autism group (see Fig. 3 , with a large $(25.7 \mathrm{~ms})$ range in the left hemisphere and a much reduced $(6.1 \mathrm{~ms})$ range in the right.

Latency variation as a function of tone frequency in early cortical ( $\sim 100 \mathrm{~ms}$ ) auditory evoked components (M100, N1) has been attributed to a combination of peripheral and central conduction delays associated with lower $(250 \mathrm{~Hz})$ vs higher $(1-6 \mathrm{kHz})$ frequency sounds [15]. Evidence in support of this view has been provided by Woods and colleagues [9], who recorded AEPs in response to tones of differing frequency $(250 \mathrm{~Hz}$ and $4000 \mathrm{~Hz})$ and reported frequency-induced latency differences with delay times that were $\sim 1 \mathrm{~ms}$ at brainstem, 3-6 ms at middle (20-50 ms) latencies, and $\sim 20 \mathrm{~ms}$ in later $(100 \mathrm{~ms})$ cortical components. Thus the absolute magnitude of frequency-induced delay appears to expand as the signal ascends in the auditory system [16]. Our findings here for control children provide indirect evidence that similar expansion functions may be present in childhood, at least in the age-range of the children tested here. Our result of a larger dynamic range in the left hemisphere for autistic children and a much-reduced dynamic range in the right hemisphere may indicate that frequency-induced expansion functions may reflect a differential maturation path in autism compared with typically developing children, and may develop differentially in the two hemispheres in children with autism.

The reduced dynamic range found in the right hemisphere for children with autism in the present investigation may relate to our previous findings, where we reported little or no evidence for age dependence in right hemisphere auditory cortical fields in autistic children [3]. If it is the case that neural conduction rates are slowed in right hemisphere 
auditory fields, then this may be a contributing factor to the present finding of a compressed dynamic range of modulation in the right hemisphere. Evidence that the right hemisphere is biased for processing spectral features in sounds, such as pitch direction, timbre, and intonation, has been provided in studies with normal and brain-damaged individuals $[17,18]$. Damage to right hemisphere auditory sites has been linked to deficits in the production and perception of the emotional contours (prosody) in spoken language [19]. If it is the case that the right hemisphere is biased for decoding spectral features in sounds, then our findings of smaller M100 latency differences in the right hemisphere in response to tonal contrasts in our sample of children with autism may imply that spectral decoding mechanisms in that hemisphere may be disrupted or impaired in autism.

Further study is needed in order to assess whether the hemispheric asymmetries reported here and in previous studies [3] replicate in other samples of autistic children and with differing stimulus parameters. While our findings here indicated that left hemisphere M100 responses were broadly similar in children with autism as compared to controls, the stimuli employed in this study (non-time-varying sinusoidal tones) contained no temporal information. A large and growing body of evidence suggests that the left hemisphere is biased for decoding rapid temporal information in sounds, such as formant transitions in speech $[17,18,20,21]$. Thus, it may be the case that simple sinusoidal tones were not sufficiently complex along a temporal dimension to produce differences in left hemisphere sites between the autistic and control children tested here. Future work employing more complex and time-varying stimuli is required in order to determine if temporally different patterns of neural activity are present early $(\sim 100 \mathrm{~ms})$ in auditory cortical processing in children with autism spectrum disorder as compared to typically developing controls.

\section{CONCLUSIONS}

While results must be treated with caution due to the small sample size and the developmental heterogeneity found in children in general and in autistic children in particular, our findings may reflect a differential path in the maturation of frequency resolution mechanisms in auditory cortex in children with autism. The reduced dynamic range found in the right hemisphere for autistic children in the present investigation may relate to our previous findings, where we reported little or no evidence for age dependence in right hemisphere auditory cortical fields in autistic children [3]. Age-related changes in the latency of auditory evoked components (such as the M100, N1) have been related to maturational changes that occur during development such as myelination, synaptogenesis, dendritic pruning, and lamination of cortical layers [22,23], with the general notion that as neural systems mature, conduction rates increase, thereby decreasing the time to peak latency in evoked components. If it is the case that neural conduction velocities are slowed in right hemisphere auditory fields in children with autism, then this may have been a contributing factor to the present finding of a compressed dynamic range of frequency induced M100 latency modulation in the right hemisphere.

\section{REFERENCES}

1. Lord C, Pickles A, McLennan J et al. J Autism Dev Disord 27, 510-517 (1997).

2. Ornitz EM. Autism at the interface between sensory and information processing. In: Dawson G, ed. Autism: Nature, Diagnosis, and Treatment. New York: Guilford Press; 1989, pp. 174-207.

3. Gage NM, Siegel B and Roberts TPL. Dev Brain Res (In Press).

4. Roberts TPL, Ferrari P, Stufflebeam SM and Poeppel D. J Clin Neurophysiol 17, 114-129 (2000).

5. Gage NM, Poeppel D, Roberts TPL and Hickok G. Brain Res 814, 236-239 (1998).

6. Gage NM, Roberts TPL and Hickok G. Cogn Brain Res 14, 303-306 (2002).

7. Roberts TPL, Ferrari P and Poeppel D. Neuroreport 9, 3265-3269 (1998).

8. Verkindt C, Bertrand O, Perrin F et al. Electroencephalogr Clin Neurophysiol 96, 143-156 (1995).

9. Woods DL, Alain C, Covarrubias D and Zaidel O. Hear Res 66, 46-52 (1993).

10. Paetau R, Ahonen A, Solomen O and Sams M. J Clin Neurophysiol 12, 177185 (1995).

11. Rojas DC, Walker JR, Sheeder JL et al. Neuroreport 9, 1543-1547 (1998).

12. Autistic Spectrum Disorders: Best Practice Guidelines for Screening, Diagnosis and Assessment. Sacramento, CA: California Department of Developmental Services; 2002.

13. American Psychiatric Association. Diagnostic and Statistical Manual of the American Psychiatric Association, 4th edn. Washington, DC: American Psychiatric Association Press; 1994.

14. Rapin I, Allen DA and Dunn MA. Developmental language disorders. In: Segalowitz SJ and Rapin I (eds). Handbook of Neuropsychology, Vol. 7. New York: Elsevier Science; 1992, pp. 111-137.

15. Jacobson GP, Ahmad BK, Moran J et al. Ear Hear 13, 3887-3895 (1992).

16. Greenberg S, Poeppel D and Roberts TPL. International Symposium on Hearing. Belton Woods Grantham, (UK) 1997.

17. Zatorre RJ and Belin P. Cerebr Cortex 11, 946-953 (2001).

18. Zatorre RJ, Belin P and Penhume VB. Trends Cogn Sci 6, 37-46 (2002).

19. Pell MD. Brain Lang 69, 161-192 (1999)

20. Poldrack RA, Temple E, Protopapas A et al. J Cogn Neurosci 13, 687-697 (2001).

21. Jancke L, Wustenberg T, Scheich H and Heinze HJ. Neuroimage 15, 733746 (2002).

22. Eggermont JJ. Electroencephalogr Clin Neurophysiol, 70, 293-305 (1988).

23. Ponton CW, Eggermont JJ, Khosla D et al. Clin Neurophysiol 113, 407-420 (2002).

Acknowledgements: We are grateful to the children and their parents for participating. This work is supported by the Medical Investigation of Neurodevelopmental Disorders (MIND) Institute (N.G.) and the National Alliance of Autism Research (T.R.). We thank Susanne Honma, R.T. and Jeff Walker, B.S. for excellent technical assistance and Cathy Hayer for help with participant recruitment. 